\title{
Three-Dimensional Visualization of Protein Expression in Mouse Brain Structures Using Imaging Mass Spectrometry
}

\author{
Anna C. Crecelius, D. Shannon Cornett, and Richard M. Caprioli \\ Mass Spectrometry Research Center and Department of Biochemistry, Vanderbilt University, Nashville, \\ Tennessee, USA
}

\author{
Betsy Williams, Benoit M. Dawant, and Bobby Bodenheimer \\ Department of Electrical Engineering and Computer Science, Vanderbilt University, Nashville, Tennessee, \\ USA
}

\begin{abstract}
We have developed a method to visualize matrix-assisted laser desorption ionization imaging mass spectrometry (MALDI IMS) data aligned with optically determinable tissue structures in three dimensions. Details of the methodology are exemplified using the 3-D reconstruction of myelin basic protein (MBP) in the corpus callosum of a mouse brain. In this procedure, optical images obtained from serial coronal sections are first aligned to each other to reconstruct a surface of the corpus callosum from segmented contours of the aligned images. The MALDI IMS data are then coregistered to the optical images and superimposed into the surface to create the final 3-D visualization. Correlating proteomic data with anatomical structures provides a more comprehensive understanding of healthy and pathological brain functions, and holds promise to be utilized in more complex anatomical arrangements. (J Am Soc Mass Spectrom 2005, 16, 1093-1099) (c) 2005 American Society for Mass Spectrometry
\end{abstract}

W ith the introduction of computer tomography (CT, positron emission tomography (PET) and magnetic resonance imaging (MRI, 3-D medical imaging has become important in studying anatomical, physiological, and functional information [1]. However, these in vivo tomographic imaging techniques do not have the ability to display protein distributions. Histochemical techniques provide 2-D maps of protein distributions, but require an antibody for visualizing each known protein, and only a limited number of proteins can be visualized in each slice using this technique. In recent years, a number of mass spectrometry techniques have been used to obtain the $\mathrm{x} / \mathrm{y}$ spatial localization of many compounds on sample surfaces [2-6]. Of the current techniques, MALDI IMS provides the widest $\mathrm{m} / \mathrm{z}$ range of species which can be imaged and is most commonly used to image protein distributions in thin sections of tissue. Using the technique, imaging is accomplished by acquiring a mass spectrum at discrete locations along a grid pattern prescribed over the surface. The resulting data set includes the $\mathrm{x}, \mathrm{y}$ coordinates (pixel) and the corresponding spectra. By extracting the measured intensity values for a particular $\mathrm{m} / \mathrm{z}$ plane from the data set and plotting on a color scale,

Published online May 31, 2005

Address reprint requests to Dr. R. M. Caprioli, Mass Spectrometry Research Center, Vanderbilt University, 465 21st Avenue S, Room 9160, Medical Research Building III, Nashville, TN 37232, USA. E-mail: r.caprioli@vanderbilt.edu
2-D ion images can be created. Expanding this technique to include images of serial sections from a single specimen provides a depth dimension to the data set, allowing the 3-D representation of even unidentified proteins in their full spatial and multi-dimensional distribution.

Different image modalities are often combined in neuroimaging to assist in the understanding of brain functions in normal and diseased stages [7-9]. Unlike in vivo tomographic imaging techniques, 2 -D modalities such as optical, histological, and MALDI IMS images require the specimen to be sliced into thin sections, which can produce tissue tearing and deformation. Therefore, correlating consecutive 2-D images to obtain a 3-D reconstruction is more demanding. In order to reconstruct a 3-D visualization involving data from different modalities, the establishment of a correspondence between the modalities through image registration techniques [10] is required.

The present study focuses on developing the 3-D visualization of proteomic data correlated to anatomical features in the brain. In the present example, we chose to model the distribution of myelin basic protein (MBP) in the corpus callosum (CC). The mouse brain was chosen for our first model because of its small size and because an atlas exists which can be used as reference for the methodology development. To create the 3-D model of MBP within the CC, optical, histological, and MALDI IMS images were acquired and processed ac- 
a) Data acquisition

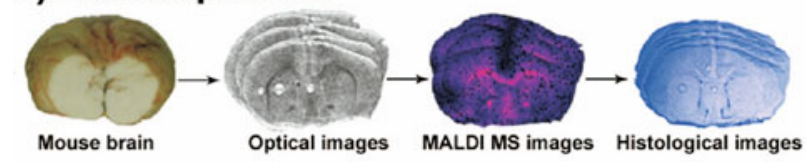

b) Data processing

i ) Inter-section registration

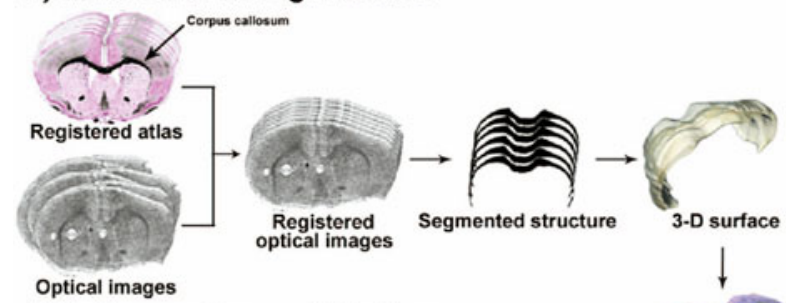

ii ) Intra-section registration

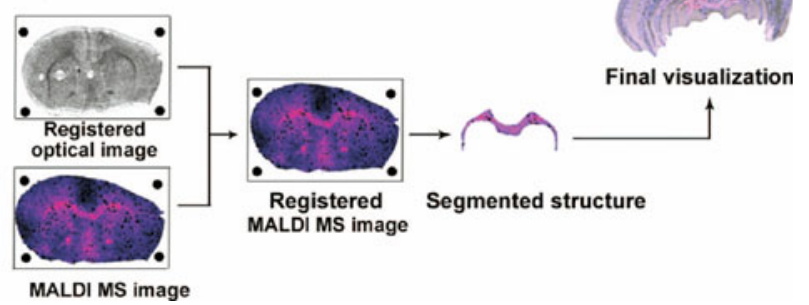

Figure 1. Steps for 3-D visualization of MALDI IMS data in a mouse brain structure. (a) Acquisition of optical, MALDI IMS, and histological images from serial sections. (bi) Registration of optical images to each other using an atlas as template followed by structure segmentation and reconstruction. (bii) Registration of MALDI IMS image to respective optical image followed by segmentation and insertion into reconstructed structure.

cording to the workflow illustrated in Figure $10^{\circ} \mathrm{A}$ number of registration steps were required to produce the final 3-D protein distribution. First, optical micrographs of unstained sections were aligned to a reference atlas. Although not explicitly necessary, use of the external reference improves the consistency of our modeling and eliminates the step of generating our own reference. Next, the optical images and MALDI IMS images were registered to each other based on landmarks visible in both imaging modalities. Finally, a 3-D surface of the anatomical feature was created from the aligned optical images and the created 2-D MALDI IMS images were inserted into the 3-D surface model according to their $\mathrm{z}$ coordinate position. The resulting 3-D model provides, for the first time, a unique correlated view of MBP distribution within the CC of mouse brain. However, the methodology can be used to map the distribution of any protein visible in the ion image spatially correlated with anatomical features observed in optical micrographs.

\section{Methods}

\section{Specimen Preparation and Cryosectioning}

The brain of a male, 6-week old C57Bl/6J mouse was removed from the skull, loosely wrapped in aluminum foil, and immediately frozen by slow immersion into liquid nitrogen for several seconds. The brain was stored at $-80{ }^{\circ} \mathrm{C}$ until sectioning in order to minimize protein degradation caused by temperature and oxidation $^{\circ}[11] .{ }^{\circ}$ The $^{\circ}$ unfixed ${ }^{\circ}$ brain $^{\circ}$ was $^{\circ}$ sectioned $^{\circ}$ at $^{\circ}-16^{\circ} \mathrm{C}$ into successive coronal sections spanning the corpus callosum using a cryostat (CM3050S Leica, Nussloch, Germany). Each section was thaw-mounted onto indium-tin oxide coated glass slides (Delta Technologies Ltd., Stillwaters, MN) and stored at $-80^{\circ} \mathrm{C}$ until time of analysis.

\section{Optical Image Acquisition}

Prior to MALDI IMS analysis, the glass slide with the desired mouse brain section was thawed in a vacuum desiccator for $15 \mathrm{~min}$ to avoid moisture condensation that could cause delocalization of proteins. Four black ink dots (diameter: $\sim 800 \mu \mathrm{m}$, printed on an adhesive label using a laser printer, were attached to the slide framing the brain slice for use as registration landmarks. An optical image of the section and surrounding landmarks was acquired using a $640 \times 480$ digital capture board coupled to an analog camera. The image resolution was $35 \mu \mathrm{m} /$ pixel and was stored to disk in tiff format.

\section{Coating Brain Section with MALDI Matrix}

Paper masking was applied over the ink spots, and the brain section was coated with MALDI matrix. The matrix (sinapinic acid, $20 \mathrm{mg} / \mathrm{ml}$ in acetonitrile/water/ TFA $50 / 50 / 0.3$, vol/vol) was deposited uniformly over the entire mouse brain section using a TLC reagent sprayer (Fisher Scientific, Suwanee, GA) with nitrogen nebulizing gas. Typically $15 \mathrm{ml}$ of matrix solution was consumed by passing the spray across the section in 15 to 20 passes from a distance of approximately $15 \mathrm{~cm}$. Following each pass, the surface of the mouse brain section was allowed to dry for up to $2 \mathrm{~min}$. Humidity and temperature were maintained at $22{ }^{\circ} \mathrm{C}$ and $50-60 \%$ during the deposition process. The crystal layer density was monitored between spray cycles by visual observation of the coated section under a microscope. When a continuous layer of matrix crystals was achieved, the coated mouse brain slice was wetted twice with $2 \mu \mathrm{l}$ of a $0{ }^{\circ} \mathrm{C}$ cold aqueous $10 \mathrm{mM}$ ammonium citrate solution to ${ }^{\circ}$ hhance ${ }^{\circ} \mathrm{MALDI}^{\circ}{ }^{\circ}{ }^{\circ}{ }^{\circ}$ intensities ${ }^{\circ}[12]$.

\section{MALDI IMS Analysis}

Mass spectra were acquired on a MALDI-TOF Voyager DE-STR mass spectrometer (Applied Biosystems, Framingham, MA) equipped with a $2 \mathrm{~Hz} 337 \mathrm{~nm}$ nitrogen laser. The laser spot size was $50 \mu \mathrm{m}$ as determined by measuring ablated spots in a thin film of $\alpha$-CHCA matrix. Data were acquired by averaging 150 laser shots per spectrum using an accelerating voltage of $25 \mathrm{kV}$ under optimum delay extraction conditions. The MS 
image acquisition is controlled by custom-developed software $\left[13,{ }^{\circ} 14\right]^{\circ}\left(\mathrm{MALDI}^{\circ} \mathrm{MS}^{\circ} \mathrm{Imaging}^{\circ} \mathrm{Tool}^{\circ},{ }^{\circ} \mathrm{MMSIT}\right.$, which interacts with the Applied Biosystems software. Each mouse brain section and its corresponding four ink spots were acquired in batch mode, without user interaction. The acquired IMS images include mass spectra only from regions of the tissue or the ink spots; intermediate areas of the plate containing no sample information were zero-filled to complete the rectangular image field. The resulting MALDI images were stored in a modified form of the Analyze data format (Mayo Foundation, Rochester, MN).

\section{Histology}

Following the MALDI IMS analysis, the mouse brain sections were Nissl stained after MALDI matrix removal. The plate with the mouse brain section was first wetted with successive aliquots of MiliQ water and $100 \%$ ethanol before immersion into a 70\% ethanol bath for $2 \mathrm{~min}$. The success of the matrix removal was determined by microscopic inspection. The staining procedure ${ }^{\circ}$ was $^{\circ}$ previously ${ }^{\circ}$ reported $^{\circ}[15] .{ }^{\circ}$ Briefly, ${ }^{\circ}$ dried sections were dehydrated in a descending series of ethanol and water, stained using Toluidine Blue $\mathrm{O}$ (0.17\% Toluidine Blue $\mathrm{O}$ in $0.2 \mathrm{M}$ acetate buffer, $\mathrm{pH}$ 4.45). Mouse brain sections were then washed in water, differentiated in an ascending series of ethanol, cleared in Histoclear (National Diagnostics, Atlanta, GA) and coverslipped using DPX (FLUKA, Milwaukee, WI). Optical images of the Nissl stains were acquired and stored as described earlier.

\section{Protein Identification}

For protein identification, two male $\mathrm{C} 57 \mathrm{Bl} / 6 \mathrm{~J}$ mouse brains (0.4 g each) were homogenized and fractionated into crude nuclear/cell membrane/cell debris pellet, crude mitochondrial/lysosomal pellet, microsomal pellet, and cytosol using sequential centrifugation, as described $^{\circ}$ previously $^{\circ}[16] . .^{\circ}$ Salt $^{\circ}$ extracts $^{\circ}$ of ${ }^{\circ}$ the ${ }^{\circ}$ nuclear pellet were obtained by suspending the extract first in $0.3 \mathrm{M} \mathrm{NaCl} / 0.01 \mathrm{M}$ Tris- $\mathrm{HCl}\left(250 \mu \mathrm{l} ; \mathrm{pH} 7.4 ; 4^{\circ} \mathrm{C}\right)$ using a motor driven polypropylene pestle, followed by centrifugation $\left(10 \mathrm{~min} ; 16,000 \times g ; 4^{\circ} \mathrm{C}\right)$. After removing the supernatant, the pellet was suspended in $2 \mathrm{M}$ $\mathrm{NaCl} / 5 \mathrm{M}$ urea/0.01 M Tris-HCl $\left(250 \mu \mathrm{l} ; \mathrm{pH} 7.5 ; 4{ }^{\circ} \mathrm{C}\right)$. Both soluble salt extracts and the cytosolic fraction were analyzed by MALDI mass spectrometry determining the fraction containing the targeted proteins. This frac-

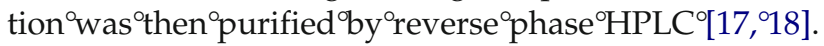
HPLC fractions containing the targeted proteins were digested with trypsin and the resulting peptides were mass analyzed and sequenced by MS and MS-MS analysis, using a 4700 Proteomics Analyzer (Applied Biosystems, Foster City, CA).

\section{Image Processing}

Processing of the MS image data was accomplished using a variety of in-house and commercial software tools. Specifically, a script was written to process each spectrum in the image file using an 11 point Gaussian smooth and the advanced baseline correction functions in the Applied Biosystems Data Explorer version 4.4 application. Protein images specific to the CC were extracted as 2-D ion density maps for each tissue section, where the intensity of the protein was plotted as a function of its $x / y$ coordinates on the tissue surface. Processing of the 2-D ion density maps was accomplished using Adobe Photoshop 7.0 and Matlab version 6.5 with the image toolbox (MathWorks). 3-D models (shown in Supplementary Video 1) were rendered in Maya from surfaces reconstructed from the segmented and $^{\circ}$ registered ${ }^{\circ}$ image $^{\circ}$ features ${ }^{\circ}$ using $^{\circ}$ fastRBF $[19]$.

\section{Results and Discussion}

\section{Data Acquisition}

Accurate registration of the optical images of serial sections requires minimal tissue tearing and deformation. Hence, a number of approaches were tested, including variation of section thickness, and the use of embedding media to minimize tissue tearing and deformation. The optimum thickness for reproducible sectioning was $20 \mu \mathrm{m}$; smaller sections tore easily, while thicker sections tended to crack and warp when dehydrated under vacuum. To minimize tissue tearing, several embedding media, such as OCT (optical cutting temperature ${ }^{\circ}$ polymer) ${ }^{\circ} 20-40 \%{ }^{\circ}$ gelatin ${ }^{\circ}$ solution $^{\circ}[20]$, Cryo-Gel (water based sucrose solution), or $2 \%$ (wt/ vol $)^{\circ}$ carboxymethylcellulose ${ }^{\circ}(\mathrm{CMC})^{\circ}$ solution ${ }^{\circ}[21]^{\circ}$ were investigated. None of the tested embedding media produced satisfactory results due to tissue deformation, and more importantly, contamination of subsequent MALDI IMS analysis. Hence, optical images of 264 consecutive $20 \mu \mathrm{m}$ thick sections spanning the CC (Bregma -3.34 to $1.94 \mathrm{~mm}$ ) were acquired from a nonembedded mouse brain. Ten of these sections, in 400-500 $\mu \mathrm{m}$ steps throughout the tissue volume, were subjected to MALDI IMS analysis followed by Nissl staining to reference the sections to a stereotaxic coordinate $^{\circ}$ system $^{\circ}[22]$.

\section{Data Processing}

Creating a 3-D visualization of proteomic data involved two steps, inter- and intra-section registration. The inter-section registration ensured the accurate alignment of optical images while the intra-section registration aligned the lower resolution MALDI IMS data to the registered optical image of the corresponding slice.

Inter-section registration. All optical images were aligned to each other in order to construct a 3-D volume of the CC. 
A number of algorithms were evaluated for registration accuracy, including alignment based on intensity values, whole brain contours, and interior feature contours. Tearing and deformation along the section boundaries made alignment based on contour matching and mutual intensity information unreliable. Interior anatomical features exhibited less distortion and sections were aligned sequentially using the contour of the CC. The contrast observed in the optical images is relatively low because of the top-down illumination required to observe the ink spots. Consequently, automated detection of the CC contour was not possible and it became necessary to segment the CC by manually defining the contour. The CC contours were segmented from the optical images and aligned to CC contours similarly ${ }^{\circ}$ segmented $^{\circ}$ from $^{\circ} \mathrm{a}^{\circ}$ mouse $^{\circ}$ brain $^{\circ}$ atlas $^{\circ}[23]$, using ${ }^{\circ} a^{\circ}$ modified ${ }^{\circ}$ iterative ${ }^{\circ}$ closest $^{\circ}$ point $^{\circ} \operatorname{method}^{\circ}[24]^{\circ}$ to compute a rigid body transformation, i.e., rotation and translation only. It should be stressed that warping or stretching was avoided here because of the tendency to "force fit" the images, causing unacceptable distortion of the low contrast images. Because sections in the reference atlas were collected at a spacing of $200 \mu \mathrm{m}$, each of the optical images was registered to the nearest atlas image using distance from Bregma as reference. Since the contour of the CC in the brain atlas exhibited minimal variations between each $200 \mu \mathrm{m}$ interval this was considered acceptable within the limits of our present spatial resolution. Because the individual atlas slices were already in registration, this technique served to align the stack of optical images. A 3-D surface of the segmented CC was calculated from the aligned optical images ${ }^{\circ}$ using ${ }^{\circ} a^{\circ}$ surface ${ }^{\circ}$ fitting ${ }^{\circ}$ tool, ${ }^{\circ}$ FastRBF $^{\circ}[19]{ }^{\circ}$ The resulting smooth surface mesh of the $\mathrm{CC}$ was then rendered ${ }^{\circ}$ using ${ }^{\circ}$ Maya $^{\circ}[25] .{ }^{\circ}{ }^{\circ}$ or ${ }^{\circ}$ sample ${ }^{\circ}$ tissues ${ }^{\circ}$ in ${ }^{\circ}$ which an external reference atlas doesn't exist, an internal reference atlas can be created by either staining alternate sections from the sample tissue or successive sections from a second tissue sample. To simplify the methodology development, the external atlas was used as reference. However, only a few of these exist and our same methodology can be applied to internal references generated by staining alternate sections of the sample tissue or successive sections from a second tissue sample.

Intra-section registration. 2-D MALDI IMS images were created for proteins of interest and registered to the corresponding optical image using landmarks visible in both images. Ion images are of relatively low resolution, producing anatomical boundaries which are not as distinct as those observed in the optical data set. As a result, we found the cumulative error produced by aligning successive ion images to be greater than that produced by aligning each ion image to its respective optical image. Intrinsic landmarks, such as small holes made through the tissue volume, were found to cause unacceptable tearing as the nonembedded tissue was sectioned. Artificial landmarks were created by framing a)

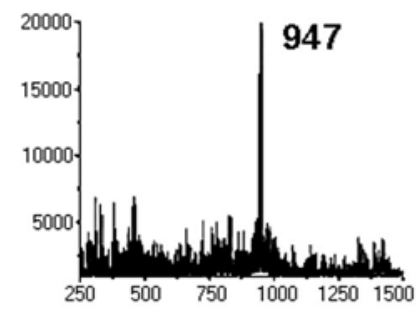

b)

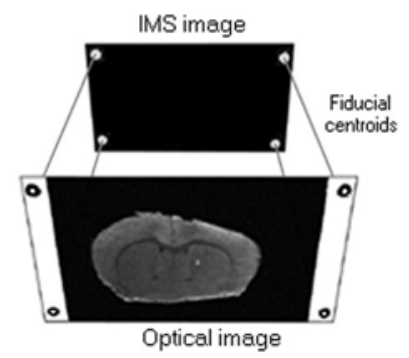

Figure 2. Intra-section registration. (a) Representative spectrum from the black toner, showing intense ion at $m / z$ 947, used to create the IMS image. (b) The centroids of the four black ink fiducials in the optical image are shown aligned to the corresponding fiducials in the IMS image.

the section with black ink spots printed on adhesive labels. The landmarks produced an intense ion image at $\mathrm{m} / \mathrm{z} 947$ by direct laser desorption, as illustrated in the $50^{\circ} \mu \mathrm{m}^{\circ}$ ion $^{\circ}$ image $^{\circ}$ shown $^{\circ}$ in $^{\circ}$ Figure $^{\circ} 2 \mathrm{a}^{\circ}$ and $^{\circ}$ were $^{\circ}$ also readily ${ }^{\circ}$ observed ${ }^{\circ}$ in $^{\circ}$ the ${ }^{\circ}$ optical ${ }^{\circ}$ image, ${ }^{\circ}$ shown ${ }^{\circ}$ in ${ }^{\circ}$ Figure 2b. ${ }^{\circ}$ An ${ }^{\circ}$ automated ${ }^{\circ}$ algorithm ${ }^{\circ}$ (see ${ }^{\circ}$ Supplementary ${ }^{\circ}$ material) determined the fiducials in both images and a transformation minimizing the root mean square distance between the centroid of the fiducials was applied. This transformation, which involves a rotation, a translation, and an isotropic scaling factor, was computed using ${ }^{\circ}$ the ${ }^{\circ}$ methods ${ }^{\circ}$ previously $^{\circ}$ described $^{\circ}[26,27] .{ }^{\circ}$ Using the relation between fiducial registration error over the $\mathrm{CC}^{\circ}$ and ${ }^{\circ}$ registration ${ }^{\circ}$ rror $^{\circ}$ over ${ }^{\circ}$ the entire ${ }^{\circ}$ image [28], the registration error of this technique was determined to be on the order of $30 \mu \mathrm{m}$. Since the fiducial centroids in the IMS image were determined by irradiating the paper surface holding the toner spots with a laser beam from a $45^{\circ}$ incident angle, an additional error of $60 \mu \mathrm{m}$ was encountered. The $60 \mu \mathrm{m}$ error was caused by a height difference of $60 \mu \mathrm{m}$ between the mouse brain section and the paper holding the toner spots. Considering both error components, the accuracy achieved by this technique is still less than the width of a single pixel in the MALDI IMS images, which is $100 \mu \mathrm{m}$. A more detailed description of the registration algorithm along with a discussion of the errors encountered with this technique can be found in the Supplementary material.

The registration process as described assumes that no shrinkage occurs during section dehydration in the mass spectrometer. To verify this, optical images from three serial sections ranging from 20 to $50 \mu \mathrm{m}$ thick were collected before and after the IMS analysis. The corresponding optical images before and after the IMS 


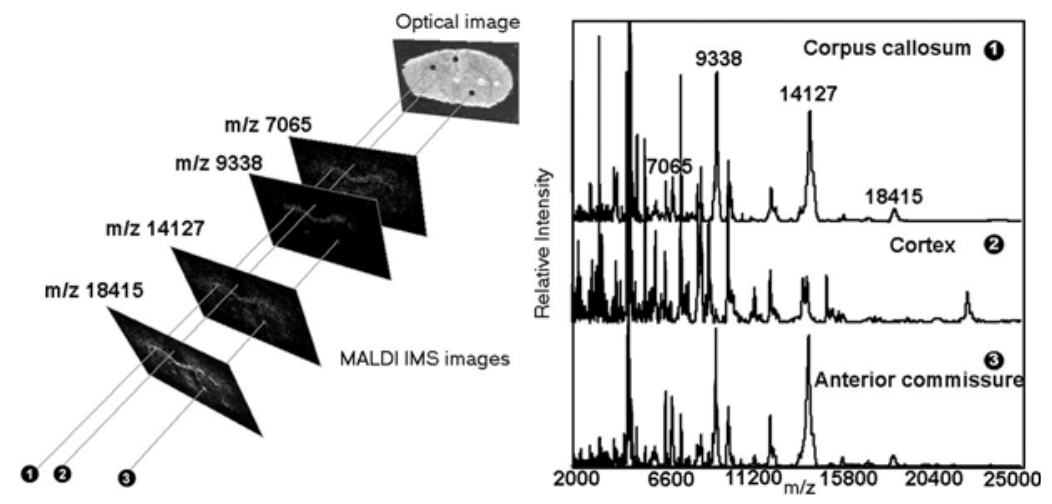

Figure 3. MALDI IMS analysis of a $20 \mu \mathrm{m}$ thick coronal mouse brain section (Bregma: $+1.32 \mathrm{~mm}$ ). The optical image of the cryosection is aligned with its corresponding MALDI IMS data. This enables the selection of specific points in the optical image, (1): corpus callosum, (2) cortex, and (3) anterior commissure, and the extraction of the corresponding mass spectra at these locations. The pixel brightness in each MALDI IMS image indicates the intensities of the following protein ions: myelin basic protein isoform 5, (m/z 18415); myelin basic protein isoform 8, (m/z 14127 and 7065); and an unidentified protein, $(\mathrm{m} / \mathrm{z}$ 9338).

analysis were registered to each other using a mutual information ${ }^{\circ}[29]^{\circ}$ based ${ }^{\circ}$ method $^{\circ}$ and $^{\circ}$ subtracted $^{\circ}$ to ${ }^{\circ}$ highlight surface area differences between the two corresponding sections. No significant tissue shrinkage was observed for all cases at the acquired optical image resolution of $35 \mu \mathrm{m}$.

Correlating anatomic structures to mass spectrometric data by intra-section registration offers some practi$\mathrm{cal}^{\circ}$ utility $^{\circ}$ as $^{\circ}$ illustrated ${ }^{\circ}$ in $^{\circ}$ Figure $^{\circ} 3 .^{\circ}$ In $^{\circ}$ this ${ }^{\circ}$ example, three locations were selected from an optical image of a $20 \mu \mathrm{m}$ coronal mouse brain section in the regions of corpus callosum, cortex, and anterior commissure. The mass spectra recorded at exactly these positions were extracted from the MALDI IMS data to highlight proteomic differences in these regions. The mass spectra show that three ions with $\mathrm{m} / \mathrm{z} 7065$ (doubly charged), 14127 (singly charged), 18415 and 9338, are highly expressed in the CC as well as in the anterior commissure. The three primary ions were identified as MBP (Swiss-Prot entry: P04370) isoform 8 and 5, respectively, by purifying the brain tissue extract, followed by proteolytic degradation. Three peptides from each protein were identified by tandem mass spectrometry (MS/MS) and protein database searching using Mascot. The ion observed at $\mathrm{m} / \mathrm{z} 9338$ has not been identified. It is important to note that previous 9 MS results [2-4, 30-32] discuss protein expression in the relative context of gross anatomical regions of the tissue sample, but with the registration methodology described here protein distributions can be attributed to cellular regions of the tissue with high specificity.

$\mathrm{A}^{\circ}$ subset ${ }^{\circ}$ of $^{\circ}$ the ${ }^{\circ}$ collected $^{\circ}$ data $^{\circ}$ set $^{\circ}{ }^{\circ}{ }^{\circ}$ shown $^{\circ}{ }^{\circ}{ }^{\circ}$ Figure 4. ${ }^{\circ}$ Five ${ }^{\circ}$ sections ${ }^{\circ}$ from ${ }^{\circ}$ the ${ }^{\circ}$ posterior ${ }^{\circ}$ to ${ }^{\circ}$ the anterior ${ }^{\circ}$ part ${ }^{\circ}$ of the brain are shown with the corresponding optical, histological, and MALDI IMS images of four different ions. The tissue region was segmented from the MALDI IMS images using the corresponding aligned optical images as template. A linear intensity scale normalized to the maximum ion intensity in the brain section was applied to create the MALDI IMS images.

Visualization. The 3-D reconstruction of MBP isoform 5 $(\mathrm{m} / \mathrm{z}$ 18415) distributed throughout the CC volume is presented ${ }^{9}{ }^{\circ}$ Figure 5 and ${ }^{\circ}$ in Movie 1 . The volume of the CC, shown in yellow, was segmented from the 264 aligned optical images. Ten MALDI IMS images were created from MBP isoform 5 using a linear color scale ranging from yellow to green representing minimum to maximum ion intensity. The MALDI IMS images were segmented along the contours of the CC in the regis-

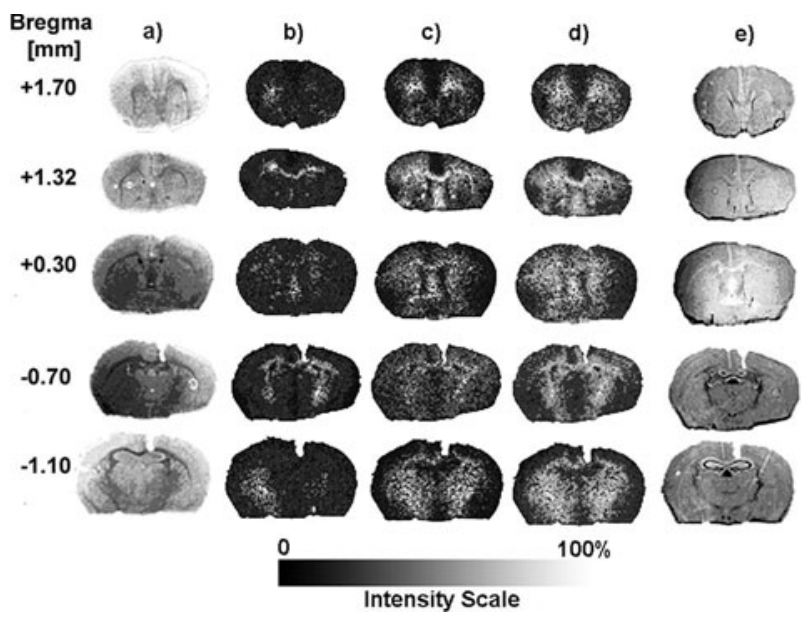

Figure 4. A sub-set of the acquired images. (a) Optical images, (b) (c) (d) MALDI IMS images corresponding to an unidentified protein, myelin basic protein isoform 5 , and myelin basic protein isoform 8, respectively, and (e) Nissl stained images acquired from the same mouse brain sections spanning Bregma -1.10 to +1.70 . The MALDI IMS images are aligned to the respective optical images and segmented at the slice boundaries using the optical image as a mask. A linear gray scale is applied to the MALDI IMS images with white pixels indicating the highest peak intensity within the ion image. 


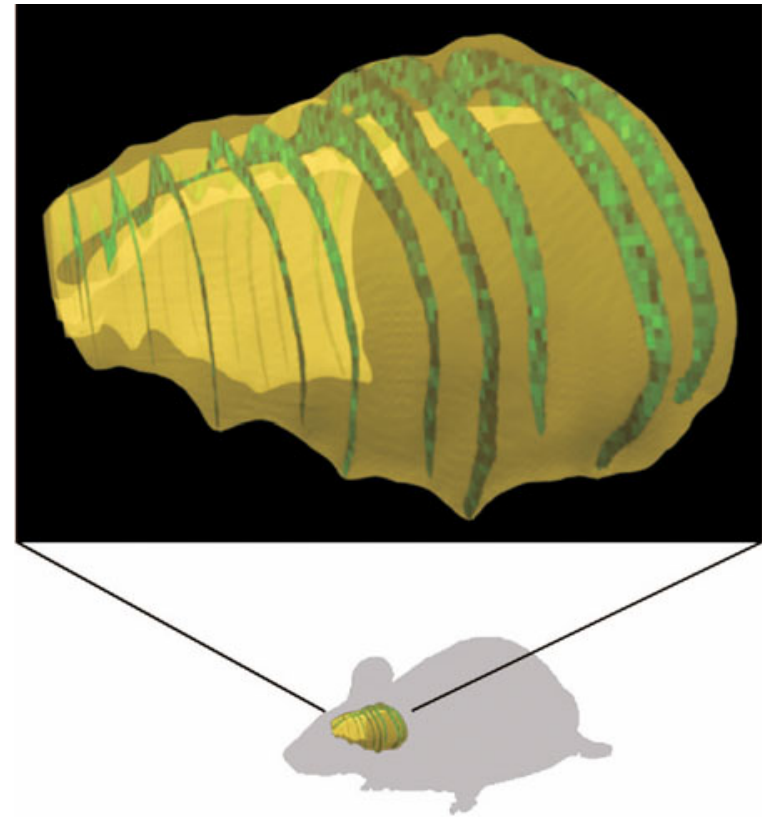

Figure 5. 3-D visualization of MALDI IMS data. The CC volume, shown in yellow, is reconstructed from 264 consecutive aligned optical contours. Ten sections equally spaced throughout the CC were analyzed by MALDI IMS and the resulting images for MBP isoform $5(\mathrm{~m} / \mathrm{z} 18415)$ are inserted into the volume. A linear green to yellow color scale is assigned with yellow showing no protein signal.

tered optical images, since MBP is present in the myelin membrane $^{\circ}[33]^{\circ}{ }^{\circ}$ which $^{\circ}$ surrounds ${ }^{\circ}$ axons $^{\circ}$ in $^{\circ}$ the ${ }^{\circ}$ brain. The segmented MALDI IMS images were inserted into the CC surface at the appropriate $\mathrm{z}$-coordinate.

Object visualization addresses questions in biology and medicine across scales, ranging from single molecules to cells, organs, and finally, to whole animal and human bodies. The main focus has primarily been to gain anatomical, physiological, and functional information in order to understand their relationship to each other ${ }^{\circ}[34]^{\circ}$ and ${ }^{\circ}$ to ${ }^{\circ}$ detect $^{\circ}$ and ${ }^{\circ}$ treat ${ }^{\circ}$ disease. ${ }^{\circ}$ The ${ }^{\circ}$ collected information is ${ }^{\circ}$ typically ${ }^{\circ}$ retrievable in ${ }^{\circ}{ }^{\circ}$ database $^{\circ}[35]^{\circ}$ or atlas $^{\circ}\left[23,{ }^{\circ} 36\right] .^{\circ}$ Developing $^{\circ}$ the $^{\circ} 3-\mathrm{D}^{\circ}$ reconstruction $^{\circ}$ of MALDI IMS data correlated to anatomical information expands the range of current imaging applications. Although imaging mass spectrometry does not produce ion distribution maps for all proteins present in the tissue, several hundred proteins can be visualized within a sample. The developed methodology can be used to correlate distributions of the observed proteins with physiological and structural information observed by in vivo imaging techniques, such as CT, PET, and MRI. Studying the degree to which protein expression influences structures and functions can build the bridge between the genome and proteome. The possibility to determine modifications of the proteins, which are not apparent from the DNA sequence, such as isoforms and post-translational modifications with MALDI-MS, and to observe the localization of these modified proteins within a tissue can enhance the understanding of their function.

\section{Conclusions}

Correlating changes to local protein distributions caused by diseases, such as cancer, Alzheimer's disease, Parkinson's disorders, with both invasive and noninvasive imaging techniques, will provide a more informative and complete understanding of these diseases. The classification of cancerous tissue based on the MALDI MS $^{\circ}$ protein $^{\circ} \operatorname{profiles}^{\circ}\left[37,{ }^{\circ} 38\right]^{\circ}$ is $^{\circ}$ one $^{\circ}$ of ${ }^{\circ}$ the ${ }^{\circ}$ growing diagnostic applications. Tumors are typically categorized by noninvasive techniques before surgery, and recently MRI combined with MR spectroscopy imaging using brain metabolite information as classifier have been $^{\circ}$ developed ${ }^{\circ}\left[39\right.$ ] $^{\circ}$ Cross-correlating $^{\circ}$ this $^{\circ}$ approach with a classification based on differentially expressed proteins employing MALDI MS would improve the diagnosis of tumors and even allow classification in all three dimensions. This would also permit the visualization of tumor heterogeneity on the proteomic level besides commonly used histological characterization and could lead to a more personalized treatment. Changes in protein expression induced by a drug candidate would also enhance the field of drug discovery. The spatial localization of drugs and the resulting changes in protein expression can give important in vivo $^{\circ}$ biological $^{\circ}$ information $^{\circ}[40] .^{\circ}$ The $^{\circ}$ metabolites $^{\circ}$ of administered drugs can be analyzed simultaneously by MALDI MS and the knowledge of their spatial localization within the organs of the dosed animal can be useful in the characterization of their toxicity.

\section{Acknowledgments}

The authors thank Michael Bubser for assistance with the histology and Ariel Deutch for helpful discussions. This research was supported by the National Institute of Health, National Cancer Institute, and National Institute on Drug Abuse.

\section{Supplementary Material}

Supplementary data associated with this article can be found, ${ }^{\circ}$ in $^{\circ}$ the $^{\circ}$ online $^{\circ}$ version, $^{\circ}$ at ${ }^{\circ}$ doi: $^{\circ}$ 10.1016/ j.jasms.2005.02.026.

\section{References}

1. Robb, R. A. Three-Dimensional Biomedical Imaging-Principles and Practice; VCH Publishers: New York, NY, 1994; pp. $1-19$.

2. Chaurand, P.; Schwartz, S. A.; Billheimer, D.; Xu, B. J.; Crecelius, A.; Caprioli, R. M. Integrating Histology and Imaging Mass Spectrometry. Anal. Chem. 2004, 76, 1145-1155.

3. Chaurand, P.; Schwartz, S. A.; Caprioli, R. M. Assessing Protein Patterns in Disease Using Imaging Mass Spectrometry. J. Prot. Res. 2004, 3, 245-252.

4. Chaurand, P.; Schwartz, S. A.; Caprioli, R. M. Profiling and Imaging Proteins in Tissue by MS. Anal. Chem. 2004, 76, 87A-93A.

5. Luxembourg, S. L.; Mize, T. H.; McDonnell, L. A.; Heeren, R. M. A. High-Spatial Resolution Mass Spectrometric Imaging of Peptide and Protein Distributions on a Surface. Anal. Chem. 2004, 76, 5339-5344. 
6. Todd, P. J.; McMahon, J. M.; McCandlish, C. A. Secondary Ion Images of the Developing Mouse Brain. J. Am. Soc. Mass Spectrom. 2004, 15, 1116-1122.

7. Ashburner, J.; Csernansky, J. G.; Davatzikos, C.; Fox, N. C.; Frisoni, G. B.; Thompson, P. M. Computer-Assisted Imaging to Assess Brain Structure in Healthy and Diseased Brains. Lancet Neurol. 2003, 2, 79-88.

8. Bjaalie, J. G. Localization in the Brain: New Solutions Emerging. Nat. Rev. Neurosci. 2002, 3, 322-325.

9. Toga, A. W.; Thompson, P. M. Maps of the Brain. Anat. Rec. 2001, 265, 37-53.

10. Toga, A. W.; Thompson, P. M. The Role of Image Registration in Brain Mapping. Image Vis. Comput. J. 2001, 19, 3-24.

11. Franzen, B.; Yang, Y.; Sunnenmark, D.; Wickmann, M.; Ottervald, J.; Oppermann, M.; Sandberg, K. Dihydroyrimidinase Related Protein-2 as a Biomarker for Temperature and Time Dependant Post Mortem Changes in the Mouse Brain Proteome. Proteomics 2003, 3, 1920-1929.

12. Zhu, Y. F.; Taranenko, N. I.; Allman, S. L.; Taranenko, N. V.; Martin, S. A.; Haff, L. A.; Chen, C. H. Oligonucleotide Sequencing by Fragmentation in Matrix-Assisted Laser Desorption/Ionization Time-of-Flight Mass Spectrometry. Rapid Commun. Mass Spectrom. 1997, 11, 897-903.

13. Stoeckli, M.; Farmer, T. B.; Caprioli, R. M. Automated Mass Spectrometry Imaging with a Matrix-Assisted Laser Desorption Ionization Time-of-Flight Instrument. J. Am. Soc. Mass Spectrom. 1999, 10, 67-71.

14. Stoeckli, M.; Staab, D.; Staufenbiel, M.; Wiederhold, K.-H.; Signor, L. Molecular Imaging of Amyloid B Peptides in Mouse Brain Sections Using Mass Spectrometry. Anal. Biochem. 2002, 311, 33-39.

15. Schroeter, S. Nissl or Counter-Staining with Toluidine Blue O; htpp://bret.mc.vanderbilt.edu/cmn/html/toluidineblue.pdf (2003).

16. Harris, E. L. V.; Angal, S. Protein Purification Methods; IRL Press: New York, 1989; pp. 1-27.

17. Chaurand, P.; DaGue, B. B.; Ma, S.; Kasper, S.; Caprioli, R. M. Strain-Based Sequence Variations and Structure Analysis of Murine Prostate Specific Spermine Binding Protein Using Mass Spectrometry. Biochemistry 2001, 40, 9725-9733.

18. Chaurand, P.; DaGue, B. B.; Pearsall, R. S.; Threadgill, D. W.; Caprioli, R. M. Profiling Proteins from AzoxymethaneInduced Colon Tumors at the Molecular Level by Matrix Assisted Laser Desorption/Ionization Mass Spectrometry. Proteomics 2001, 1, 1320-1326.

19. Carr, J. C.; Beatson, R. K.; Cherrie, J. B.; Mitchell, T. J.; Fright, W. R.; McCallum, B. C.; Evans, T. R. Reconstruction and Representation of 3D Objects with Radial Basic Functions; ACM SIGGRAPH: Los Angeles, CA, 2001; pp 67-76.

20. Klekamp, J.; Ridel, A.; Kretschmann, H. J. A New Embedding and Sectioning Technique (Macrovibratome) for Macroscopic and Morphometric Examination Especially of the Human Brain. Hirnforsch. 1985, 26, 33-40.

21. Toga, A. W.; Ambach, K.; Quinn, B.; Hutchin, M.; Burton, J. S. Postmortem Anatomy from Cryosectioned Whole Human Brain. J. Neurosci. Methods 1994, 54, 239-252.

22. Paxinos, G.; Franklin, K. B. J. The Mouse Brain in Stereotaxic Coordinates; Academic Press: New York, NY, 2001.
23. Sidman, R. L., Kosaras, B., Misra, B., Senft, S. High Resolution Mouse Brain Atlas; http://www.hms.harvard.edu/research/ brain (2004).

24. Besl, P. J.; McKay, N. D. A Method for Registration 3-D Shapes. IEEE Trans. Patt. Anal. Mach. Intell. 1992, 14, 239-256.

25. Lammers, J. Maya 5 Fundamentals; New Riders: Indianapolis, IN, 2003, pp. 27-50.

26. Schonemann, P. H.; Carroll, R. M. Fitting One Matrix to Another under Choice of a Central Dilation and a Rigid Motion. Psychometrica 1970, 35, 245-255.

27. Arun, K. S.; Huang, K. S.; Blostein, S. D. Least-Squares Fitting of Two 3-D Point Sets. IEEE Trans. Patt. Anal. Mach. Intell. 1987, 9, 698-700.

28. Fitzpatrick, J. M.; West, J.; Maurer, C. R. Predicting Error in Rigid-Body Point-Based Registration. IEEE Trans. Med. Imaging 1998, 17, 694-702.

29. Maes, F.; Collignon, A.; Vandermeulen, D.; Marchal, G.; Suetens, P. Multimodality Image Registration by Maximization of Mutual Information. IEEE Trans. Med. Imaging 1997, 16, 187-198.

30. Stoeckli, M.; Chaurand, P.; Hallahan, D. E.; Caprioli, R. M. Imaging Mass Spectrometry: A New Technology for the Analysis of Protein Expression in Mammalian Tissues. Nat. Med. 2001, 7, 493-496.

31. Chaurand, P.; Fouchecourt, S.; DaGue, B. B.; Xu, B. J.; Reyzer, M. L.; Orgebin-Crist, M.-C.; Caprioli, R. M. Profiling and Imaging Proteins in the Mouse Epididymis by Imaging Mass Spectrometry. Proteomics 2003, 3, 2221-2239.

32. Chaurand, P.; Schwartz, S. A.; Caprioli, R. M. Imaging Mass Spectrometry: A New Tool to Investigate the Spatial Organization of Peptides and Proteins in Mammalian Tissue Sections. Curr. Opin. Chem. Biol. 2002, 6, 676-681.

33. Kursula, P. The Current Structural Studies on Proteins of the Myelin Sheath. Int. J. Mol. Med. 2001, 8, 475-479.

34. Robb, R. A. Three-Dimensional Visualization in Medicine and Biology; Academic Press: San Diego, CA, 2000; pp. 685-712.

35. Spritzer, V. M.; Whitlock, D. G. The Visible Human Dataset: The Anatomical Platform for Human Simulation. Anat. Record (New Anat.) 1998, 253, 49-57.

36. Roland, P. E.; Zilles, K. Brain Atlas-A New Research Tool. Trends Neurosci. 1994, 17, 458-467.

37. Schwartz, S. A., Weil, R. J., Thompson, R. C., Shyr, Y., Moore, J., Johnson, M. D., Caprioli, R. M. Proteomic-Based Prognosis of Brain Tumor Patients Using Direct-Tissue MALDI Mass Spectrometry, unpublished.

38. Yanagisawa, K.; Shyr, Y.; Xu, B. J.; Massion, P. P.; Larsen, P. H.; White, B. C.; Roberts, J. R.; Edgerton, M.; Gonzalez, A.; Nadaf, S.; Moore, J. H.; Caprioli, R. M.; Carbone, D. M. Proteomic Patterns of Tumor Subsets in Non-Small-Cell Lung Cancer. Lancet 2003, 362, 433-439.

39. Simonetti, A. W.; Melssen, W. J.; van der Graaf, M.; Postma, G. J.; Heerschap, A.; Buydens, L. M. C. A Chemometric Approach for Brain Tumor Classification Using Magnetic Resonance Imaging and Spectroscopy. Anal. Chem. 2003, 75, 5352-5361.

40. Reyzer, M. L.; Hsieh, Y. S.; Ng, K.; Korfmacher, W. A.; Caprioli, R. M. Direct Analysis of Drug Candidates in Tissue by Matrix-Assisted Laser Desorption/Ionization Mass Spectrometry. J. Mass Spectrom. 2003, 38, 1081-1092. 